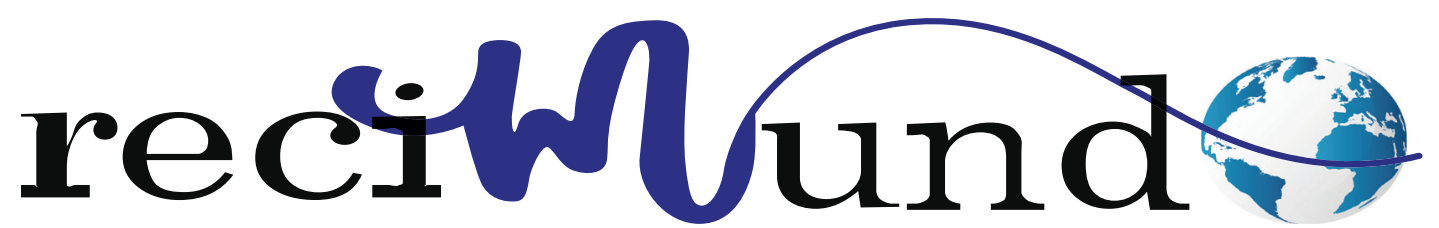

Revista Científica Mundo de la Investigación y el Conocimiento

DOI: 10.26820/recimundo/5.(2).julio.2021.52-65

URL: https://recimundo.com/index.php/es/article/view/1247

EDITORIAL: Saberes del Conocimiento

REVISTA: RECIMUNDO

ISSN: 2588-073X

TIPO DE INVESTIGACIÓN: Artículo de investigación

CÓDIGO UNESCO: 32 Ciencias Médicas

PAGINAS: $52-65$

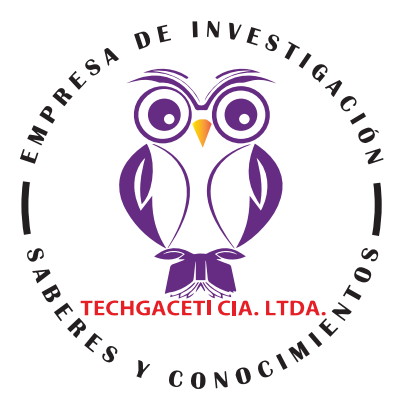

\title{
Dexametasona o hidrocortisona intravenosa como profilaxis de cefalea postpunción dural
}

Dexamethasone or intravenous hydrocortisone as prophylaxis of postdural puncture headache

Dexametasona ou hidrocortisona intravenosa como profilaxia da cefaleia pós-punção dural

Marco Antonio Calle Gómez'; Nidia Devonish²; Ingrid Esmeralda Gurumendi España3;

Maritza Alexandra Borja Santillán ${ }^{4}$

RECIBIDO: 11/04/2021 ACEPTADO: 15/06/2021 PUBLICADO: 30/07/2021

1. Magister en Derecho Civil y Procesal Civil; Especialista en Cirugía General; Especialista en Anestesiología; Diploma Superior en Docencia Universitaria; Doctor en Medicina y Cirugía; Abogado de los Tribunales y Juzgados de la Republica; Docente Facultad de Ciencias Médicas Universidad de Guayaquil, Guayaquil, Ecuador; marco.calleg@ug.edu.ec (DD https:// orcid.org/0000-0002-2706-1554

2. Doctora en Ciencias Médicas; Especialista en Anestesiología; Profesora Titular de LUZ; Venezuela; nidia.devonish@gmail. com (iD) https://orcid.org/0000-0002-4585-5374

3. Magister en Gerencia y Administración en Salud; Medico; Universidad de Guayaquil; Guayaquil, Ecuador; ingrid.grumendie@ug.edu.ec; ID https://orcid.org/0000-0001-6692-719X

1. Doctora en Educación; Magister en Gerencia de Salud para el Desarrollo Local; Especialista en Gerencia y Planificación Estratégica en Salud; Diploma Superior en Desarrollo Local y Salud; Magister en Emergencias Médicas; Medico; Licenciada en Ciencias de la Educación mención Lengua Inglesa y Lingüística; Docente Universidad de Guayaquil, Facultad de Ciencias Médicas, Guayaquil, Ecuador; iD https://orcid.org/0000-0002-2803-4662

\section{CORRESPONDENCIA \\ Marco Antonio Calle Gómez \\ marco.calleg@ug.edu.ec \\ Guayaquil, Ecuador}

() RECIMUNDO; Editorial Saberes del Conocimiento, 2021 


\section{RESUMEN}

El objeto de este artículo es determinar la eficacia de dexametasona o hidrocortisona endovenosa como profilaxis de cefalea postpunción dural en pacientes intervenidos quirúrgicamente de enero a diciembre de 2018. Fue una investigación experimental, descriptiva, prospectiva, transversal y correlacional. La muestra de pacientes electivos ( $n=60)$ se dividió en tres grupos (A: control $(n=20)$, B: Hidrocortisona 500mg EV $(n=20)$ y C: Dexametasona 16mg EV $(n=20)$ ). Estadística tipo descriptiva. Resultados: estadísticamente, los grupos en cuánto a edad (A: 66; B: 57y C: 44 ), peso (A: 68 kg; B: 67 y C: 57) y talla (A: $158 \mathrm{cms}$; B: 155 y C: 168) fueron similares; se usó en todas las pacientes aguja Sprotte de calibre 25G, con riesgo ASA I; en el grupo control (A) se observaron en el 10\% de los pacientes (2) síntomas de CPPD: cefalea ortostática, cefalea bilateral, rigidez de nuca, tinnitus, fotofobia, diplopía, náuseas, vómitos, mareos y realización de punción lumbar previa. En el Grupo de Hidrocortisona (B) no se observaron síntomas de CPPD y en el grupo dexametasona (C) no se observaron síntomas de CPPD. El promedio de aparición de síntomas fue entre 19 a 28 horas después, sin ninguna reacción adversa. Conclusiones: el empleo de ambos medicamentos tiene la misma efectividad como profilaxis de la cefalea postpunción dural.

Palabras clave: Cefalea postpunción dural; profilaxis; hidrocortisona; dexametasona; pacientes gestantes; anestesia conductiva.

\section{ABSTRACT}

Objective: to determine the efficacy of intravenous dexamethasone or hydrocortisone as prophylaxis of post-dural puncture headache in patients who underwent surgery from January to December 2018. It was an experimental, descriptive, prospective, cross-sectional and correlational investigation. The sample of elective patients $(n=60)$ was divided into three groups (A: control $(n=20)$, B: Hydrocortisone 500mg EV $(n=20)$ and C: Dexamethasone 16mg EV $(n=20))$. Descriptive statistics. Results: statistically, the groups in terms of age (A: 66; B: 57 and C: 44), weight (A: 68 kg; B: 67 and C: 57 ) and height (A: $158 \mathrm{~cm}$; B: 155 and C: 168) were similar; A 25G Sprotte needle, with ASA I risk, was used in all patients; In the control group (A), symptoms of CPPD were observed in 10\% of the patients: orthostatic headache, bilateral headache, neck stiffness, tinnitus, photophobia, diplopia, nausea, vomiting, dizziness and previous lumbar puncture. . In the Hydrocortisone Group (B) no CPPD symptoms were observed and in the dexamethasone group (C) no CPPD symptoms were observed. The average appearance of symptoms was between 19 to 28 hours later, without any adverse reaction. Conclusions: the use of both drugs is equally effective as a prophylaxis of post-dural puncture headache.

Keywords: Post-dural puncture headache; prophylaxis; hydrocortisone; dexamethasone; pregnant patients; conductive anesthesia.

\section{RESUMO}

Objetivo: determinar a eficácia da dexametasona ou hidrocortisona intravenosa na profilaxia da cefaléia pós-punção dural em pacientes operados de janeiro a dezembro de 2018. Foi uma investigação experimental, descritiva, prospectiva, transversal e correlacional. A amostra de pacientes eletivos $(n=60)$ foi dividida em três grupos $(A$ : controle $(n=20)$, B: Hidrocortisona 500mg EV $(n=20)$ e C: Dexametasona 16mg EV $(n=20))$. Estatísticas descritivas. Resultados: estatisticamente, os grupos em termos de idade (A: 66; B: 57 e C: 44), peso (A: 68 kg; B: 67 e C: 57) e altura (A: 158 cm; B: 155 e C: 168) eram semelhantes; Uma agulha Sprotte $25 \mathrm{G}$, com risco ASA I, foi usada em todos os pacientes; No grupo controle (A), sintomas de DPFC foram observados em 10\% dos pacientes: cefaleia ortostática, cefaleia bilateral, rigidez de nuca, zumbido, fotofobia, diplopia, náuseas, vômitos, tontura e punção lombar prévia. . No Grupo Hidrocortisona (B) não foram observados sintomas de DPFC e no grupo dexametasona (C) não foram observados sintomas de DPFC. O aparecimento médio dos sintomas foi entre 19 a 28 horas depois, sem qualquer reação adversa. Conclusões: o uso de ambas as drogas é igualmente eficaz como profilaxia da cefaleia pós-punção dural.

Palavras-chave: Cefaleia pós-punção dural; profilaxia; hidrocortisona; dexametasona; pacientes grávidas; anestesia condutiva. 


\section{Introducción}

La anestesia subaracnoidea tiene sus inicios en el año 1899 en las publicaciones del cirujano alemán August Bier (18611949) y unos meses más tarde por el cirujano francés Theódore Tuffier (1861-1929). La publicación de estos trabajos en revistas de gran difusión y su presentación en el XIII Congreso internacional de Medicina en Paris en Agosto de 1900 hacen que pronto cirujanos de todo el mundo ensayen estas técnicas.

En pleno siglo XXI, la anestesia subaracnoidea es técnica de elección en numerosos procedimientos quirúrgicos, ya que brinda una anestesia y analgesia segura. Así mismo el cirujano August Bier fue quien describió una de las complicaciones más frecuentes durante la práctica de este tipo de anestesia, se habla de la cefalea postpunción dural (CPPD) en el año 1898. (Gildardo $R, 2016)$, con una incidencia reportada entre el $50 \%$ al $60 \%$ a comienzos del siglo XX.

En un adulto normal la producción diaria de LCR es de $0.3 \mathrm{ml} / \mathrm{min}$ y el volumen total es de $150 \mathrm{ml}$, el cual está dividido en proporciones similares entre la bóveda craneana y la región espinal. (Torres Zafra J, 2008). Aunque no se han comprendido completamente los mecanismos fisiopatológicos que desencadenan la CPPD, se cree que la pérdida de LCR a través de la brecha creada en la duramadre, genera una disminución de la presión intracraneal. La hipotensión en el espacio subaracnoideo, produce una tracción de las meninges, el encéfalo y los nervios craneales hacia caudal, desencadenando los signos y síntomas característicos de esta condición; a su vez, según la hipótesis de Monro-Killie ocurriría además una dilatación compensatoria de las venas intracraneales (López Correa, Garzón Sánchez, Sánchez Montero, \& Muriel Viloria, 2011).
La CPPD es una complicación secundaria a la punción lumbar en anestesia subaracnoidea o por ruptura accidental de la duramadre en procedimientos de anestesia o analgesia epidural. En la historia natural de la enfermedad, los síntomas están relacionados con la pérdida de líquido cefalorraquídeo (LCR) a través de la duramadre.

En un adulto normal la producción diaria de LCR es de $0.3 \mathrm{ml} / \mathrm{min}$ y el volumen total es de $150 \mathrm{ml}$, el cual está dividido en proporciones similares entre la bóveda craneana y la región espinal (Torres Zafra, 2008). Aunque no se han comprendido completamente los mecanismos fisiopatológicos que desencadenan la CPPD, se cree que la pérdida de LCR a través de la brecha creada en la duramadre, genera una disminución de la presión intracraneal. La hipotensión en el espacio subaracnoideo, produce una tracción de las meninges, el encéfalo y los nervios craneales hacia caudal, desencadenando los signos y síntomas característicos de esta condición; a su vez, según la hipótesis de Monro-Killie ocurriría además una dilatación compensatoria de las venas intracraneales (López Correa, Garzón Sánchez, Sánchez Montero, \& Muriel Viloria, 2011).

La cefalea se presenta 24 a 72 horas después de la punción dural, aunque puede ocurrir de inmediato. La rapidez de inicio de los síntomas se relaciona con la cantidad de LCR que se pierde durante y después de la punción. El dato característico es la naturaleza postural de la cefalalgia, que se agrava en la posición erecta y se alivia al adoptar la posición supina. Clásicamente es de tipo punzante. Si no puede desencadenarse una cefalalgia por este cambio de posición, debe cuestionarse el diagnóstico (López Correa, Garzón Sánchez, Sánchez Montero, \& Muriel Viloria, 2011).

La cefalalgia puede asociarse con náuseas $(60 \%)$, vómitos (24\%), síntomas oculares (13\%), fotofobia, diplopía, dificultades 
para la acomodación, y síntomas auditivos (12\%), pérdida de la audición, hiperacusia y tinnitus. (García A. y Cols. 2005). Aunque muy rara siempre que exista la diplopía debe considerarse paresia del nervio motor ocular externo, y se cree que se origina por tracción mecánica del nervio relacionado con un decremento del volumen de LCR intracraneal. Este es el par craneal (VI) que con más frecuencia se ve afectado debido al recorrido intracraneal (López Correa, Garzón Sánchez, Sánchez Montero, \& Muriel Viloria, 2011).

Evidencia científica reporta que la CPPD se presenta en mayor proporción en mujeres que en hombres, eso denota un componente hormonal que juega un papel importante, teniendo mayor prevalencia en mujeres entre los 20 y los 40 años de edad. Con el avance de la anestesia regional ha disminuido el porcentaje de CPPD eso se debe a la introducción de agujas de menor calibre. La cefalea puede estar relacionada con el calibre y diseño de la aguja, la experiencia de quien realiza el procedimiento, la edad y el sexo del paciente.

En el tratamiento de la CPPD no existe un acuerdo universal, pero incluye desde reposo en posición supina, aines, cafeína, hasta llegar a procedimientos durales como es el parche hemático. No dejando de lado el uso de esteroides los cuales por un mecanismo no dilucidado aún con exactitud ayudan al manejo de esta patología.

Hablando de la analgesia obstétrica por punción dural; si consideramos la esperanza combinada de vida de la mujer y su hijo es de110 a 130 años. El nacimiento de un hijo sano de una madre y un padre felices representa una inversión importante en el futuro. Por lo tanto, los anestesiólogos obstetras tienen una responsabilidad particular por la salud y el bienestar de su comunidad. La obstetricia y la anestesia obstétrica son ambientes médicos y médico-legales de alto riesgo (Rendon Mejías, 2002).
Puede hablarse de 2 grandes aliados en el manejo de la CPPD como lo son la hidrocortisona y la dexametasona por vía endovenosa, cuya evidencia científica revela que previene de alguna manera la aparición de esta complicación. Aunque no se ha dilucidado exactamente el mecanismo por el cual reducen la CPPD, se han propuesto varios mecanismos:

a) el efecto antiinflamatorio directo de estos, al disminuir la producción de citoquinas proinflamatorias como la interleucina 1 (IL1), interleucina 2 (IL-2) y factor de necrosis tumoral alfa (TNF- $\square$ ),

b) actuación sobre la bomba de Sodio-Potasio $(\mathrm{Na} / \mathrm{K})$ disminuyendo la producción del LCR en los plexos coroideos y por ende disminuyendo la fuga (Sánchez González, 2010) y haciendo el efecto contrario,

c) haciendo que se retenga sal y agua y que aumente el volumen vascular y la producción de LCR (Marrón Peña \& Mille Loera, 2013).

Estos dos esteroides son la punta de lanza en el tratamiento farmacológico de la CPPD en obstetricia debido a la poca frecuencia de efectos adversos y su alta inocuidad en la lactancia materna, así como también la posibilidad de brindarle a la madre la facilidad de atender al recién nacido y por ende disminuir otros efectos como la depresión post parto (Moral Turiel, Rodríguez Simon, Sahagun de la Lastra, \& Yuste Pascual, 2002) y adicionar sus efectos estimulantes del sistema inmunológico a corto plazo.

Los conceptos y términos básicos para la investigación en el tema, pueden ser los siguientes:

AINE: Siglas que significan: Anti Inflamatorio No Esteroideo. Definición asignada a un variado grupo de diferentes composiciones químicas de medicamentos antiinflamatorios, analgésicos y antipiréticos (antitérmico), sin inhibidores a diferentes de niveles de los mediadores de la cadena inflamato-

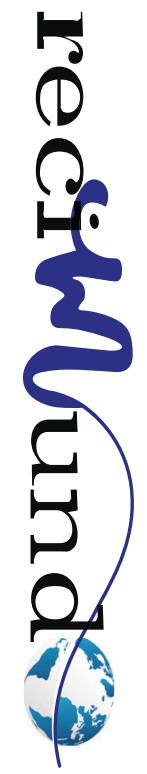


ria, como por ejemplo la enzima ciclooxigenasa (linea y salud, 2021).

Autólogo: se refiere a los elementos que constituyen el cuerpo tales como las células o los tejidos, que son propios de un individuo (Oceano-Mosby, 2001).

Cafeína: es un alcaloide blanco que pertenece al grupo de las xantinas, su apariencia física es sólida cristalina, blanca y al gusto es de sabor amargo, en tanto, entre sus acciones principales se cuentan la de actuar como un estimulante del sistema nervioso central y del corazón. Sustancia de uso extendido, presente en diversas bebidas como café, té, bebidas cola y remedios.

Ciclopentanoperhidroxifenantreno: (también Ilamado ciclopentanoperhidrofenantremo, esterano o gonano) es un hidrocarburo policíclico que se puede considerar un producto de la saturación del fenantreno asociado a un anillo de ciclopentano. Es la estructura precursora de las hormonas esteroideas y demás esteroides sintéticos. En los animales, está presente en forma de colesterol, y a partir de la acción ezimática se transforma en las diferentes hormonas esteoideas (linea y salud, 2021).

Espacio Subaracnoideo: Tejido o espacio situado por debajo de la membrana aracnoidea de las meninges y por encima de la piamadre.

Intratecal: Describe el espacio lleno de líquido entre las capas delgadas de tejidos que cubren el cerebro y la médula espinal. Se pueden inyectar medicamentos dentro del líquido o se puede extraer una muestra del líquido para someterla a prueba. Diccionario de Cáncer. Instituto Nacional de cáncer USA.

Meninges: Membranas que recubren el cerebro y la médula espinal. Las meninges están formadas por tres membranas: 1) la piamadre, que es la más interna y la que está en contacto con el cerebro; 2) la membrana aracnoidea, en situación intermedia; 3) la duramadre, la membrana más externa. Osmolaridad: Se conoce como osmolaridad a la medida que expresa el nivel de concentración de los componentes de diversas disoluciones. El concepto deriva de la presión osmótica que cambia en las células del organismo cuando se introduce la disolución en cuestión (Oceano-Mosby, 2001).

Tomando en cuenta los factores epidemiológicos, biológicos y psicosociales de las pacientes puérperas cabe entonces plantearse la siguiente interrogante: ¿será más eficaz la dexametasona o la hidrocortisona en el tratamiento profiláctico de la cefalea postpunción dural?

Para responder a estas interrogantes, se plantea el objetivo general de determinar la eficacia de la dexametasona o la hidrocortisona endovenosa como profilaxis en el tratamiento de la cefalea postpunción dural en pacientes intervenidos quirúrgicamente en el Hospital Jacobo y María Elena Ratinoff de la Ciudad de Guayaquil-Ecuador de enero a diciembre de 2018.

Igualmente, en este artículo se tratará de describir específicamente el cuadro clínico de cefalea postpunción dural, con parámetros clínicos y criterios específicos para su diagnóstico, así como analizar la eficacia de la dexametasona 16 mg EV y de hidrocortisona 500 mg EV previo al acto quirúrgico como profilaxis de la cefalea postpunción dural, comparar la incidencia de cefalea postpunción dural en cada uno de los grupos de estudio e identificar los posibles efectos adversos de la aplicación de ambos esteroides en el tratamiento profiláctico de cefalea postpunción dural.

\section{Metodología}

La presente investigación se realizó en el período entre enero y diciembre de 2018, en pacientes sometidos a intervención qui- 
rúrgica, donde se aplicó anestesia mediante punción dural de forma electiva, que fueron atendidos en el servicio de cirugía del Hospital Jacobo y María Elena Ratinoff de la ciudad de Guayaquil, Ecuador.

La definición corresponde a los parámetros mediante los cuales se medirá la aparición de la CPPD, así como también la intensidad del dolor, y el seguimiento que se le hará a los pacientes incluidos en el estudio.

Objetivo General: Determinar la eficacia de la dexametasona o la hidrocortisona endovenosa como profilaxis en el tratamiento de la cefalea postpunción dural en pacientes gestantes atendidas en la Maternidad "Armando.

\section{Tipo de investigación}

Se realizó una investigación experimental.

\section{Diseño de la investigación}

El diseño de esta investigación fue considerado de tipo prospectivo, transversal y correlacional.

\section{Población y muestra}

La población objeto de estudio estuvo representada por los pacientes quirúrgicos sometidos a procedimientos anestésicos conductivos de manera electiva en el Hospital Jacobo y María Elena Ratinoff, dentro del período comprendido entre enero y diciembre de 2018. No se tomaron muestras conformándose un censo estadístico donde se tomaron todas las unidades de investigación.

Criterios de inclusión:

Pacientes quirúrgicos sometidos a procedimientos de anestesia conductiva electiva ASA I y ASA II.

Criterios de exclusión:

- Pacientes sometidos a procedimientos de anestesia conductiva ASA III y ASA
IV.

- Pacientes que no acepten participar en el estudio.

\section{Técnicas de recolección de datos}

La técnica de recolección de datos se llevó a cabo a través de un formulario estandarizado (anexo), al cual se le practicó la validez de su contenido a través del juicio de dos expertos en la materia, donde se incluyeron: características generales (edad, peso, talla), Presencia de síntomas y signos descritos individualmente, tiempo de aparición de CPPD e identificación del grupo experimental, seguimiento del paciente y aparición de algún efecto adverso del tratamiento profiláctico. Los resultados se tabularon y registraron en cada formulario y se aplicaron pruebas de confiabilidad.

Procedimientos

A todos los participantes se les explicó el procedimiento y solicitó voluntariamente su consentimiento informado para su inclusión en el protocolo de estudio; se procedió al acto anestésico, en donde dependiendo del grupo de estudio al que se asignó cada paciente, se indicó y cumplió la dosis de profilaxis experimental, y se hizo seguimiento postoperatorio hasta el 10mo día evaluando la aparición de CPPD.

Los pacientes $(n=60)$ fueron distribuidas en tres grupos pareados: grupo A (control) $(n=20)$, grupo $B$ (profilaxis con hidrocortisona 500mg EV) $(n=20)$, grupo c (profilaxis con dexametasona 16mg EV) $(n=20)$ siendo el monitoreo idéntico para todos los grupos. Se dio inicio al acto anestésico con anestesia tipo conductiva con aguja \#25G inmediatamente se colocó dosis profiláctica según grupo. Se hizo seguimiento al postoperatorio hasta el 10mo día evaluando la aparición de CPPD la cual se definió según criterios clínicos ya descritos y se reportaron resultados de cada grupo de estudio para realizar el procedimiento estadístico correspondiente. Las primeras 24 horas el

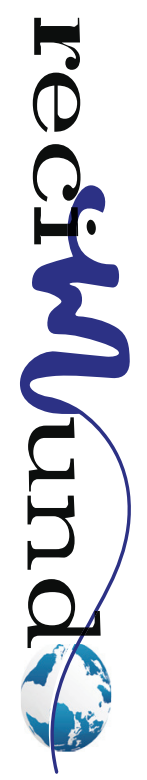


médico residente tuvo a cargo el registro de aparición de CPPD en los pacientes de estudio; desde el segundo al décimo día el anestesiólogo investigador vía whatsapp, mensaje de texto o llamada telefónica tuvo comunicación días 2, 4, 6, y 8 del postoperatorio con el paciente o familiar en el registro de aparición de CPPD, para el día 10 que fue el de retiro de puntos de la herida quirúrgica se tuvo la última entrevista con los pacientes; en el caso de que coincidió con día de feriado pasó la entrevista para el día 11 o 12 según correspondió.

\section{Técnica de análisis}

Los resultados obtenidos fueron tabulados y analizados a través del Programa estadístico SPSS versión 19, ejecutándose un tratamiento estadístico de tipo descriptivo; a saber, la presencia de signos y síntomas que definen el diagnóstico de CPPD, la intensidad del dolor y las reacciones adversas al tratamiento profiláctico se expresaron mediante frecuencias absolutas y relativas, medidas de tendencia central (medias) y de dispersión (desviación estándar). Para realizar la comparación de los resultados obtenidos entre los grupos de pacientes a evaluar, se indicaron porcentajes y se utili- zó la T de Student para datos cuantitativos; ambas con un nivel de confianza del 95\% y significancia de $p<0,05$, los resultados se expresaron en cuadros y gráficos.

\section{Resultados}

La muestra constó de 60 pacientes divididos en tres grupos, siendo un estudio experimental, uno de los grupos constó de un grupo control (A), al cual se aplicó la técnica anestésica ya descrita, sin aplicar ningún tipo de profilaxis a la técnica; y dos grupos experimentales, al primero de los cuales se le aplicó la profilaxis con Hidrocortisona 500 mg EV (B), y el otro grupo se le aplicó la profilaxis con Dexametasona $16 \mathrm{mg}$ EV (C); y se evaluó la aparición o no de CPPD en todos los grupos.

Para eliminar las posibles alteraciones que se produzcan en los grupos con la aparición de CPPD, el tipo de aguja (Sprotte) y su calibre (25G) es el mismo en todas los pacientes, de todos los grupos, todos fueron ASA I - II y a todos les fue practicada la intervención quirúrgica. Estadísticamente, los grupos en cuánto a edad, peso, talla y duración de intervención quirúrgica fueron similares, como se demuestra en la tabla 4.

Tabla 1. Datos de los grupos

\begin{tabular}{|c|c|c|c|c|}
\hline & $\begin{array}{l}\text { Edad (años) } \\
\text { Rango } \\
\text { (M+DE) }\end{array}$ & $\begin{array}{c}\text { Peso }(\mathrm{kg}) \\
\text { Rango }(\mathrm{M}+\mathrm{D}) \mathrm{E})\end{array}$ & $\begin{array}{c}\text { Talla (cms) } \\
\text { Rango }(\mathrm{M}+\mathrm{DE})\end{array}$ & $\begin{array}{l}\text { Duración de la } \\
\text { Intervención } \\
\text { (min) } \\
\text { Rango (M+DE) }\end{array}$ \\
\hline Grupo A & $(29.47,59.83)$ & $(58.99,77.2)$ & $(149.01,166.09)$ & $(44.87,78.13)$ \\
\hline Grupo B & $(28.07,56.03)$ & $(54.14,76.06)$ & $(148.28,162.92)$ & $(36.57,101.43)$ \\
\hline Grupo C & $(29.04,57.96)$ & $(56.03,79.17)$ & $(149.13,162.25)$ & $(41.93,78.07)$ \\
\hline
\end{tabular}

Fuente: Calle (2018) 
Se puede observar en que en el grupo A (control) en cuanto al dato de la edad, se tiene un rango de 29.47 años con $M+D E$ de 59.83; en cuanto al peso se encuentra un rango entre 58.99 con $\mathrm{M}+\mathrm{DE}$ de 77.2 El parámetro Talla, se puede observar con un rango entre $149.01 \mathrm{cms}$ con $\mathrm{M}+\mathrm{DE}$ de 166.09 y la duración de la intervención quirúrgica, expresada en Minutos, tiene un rango de entre 44.87 min con $M+D E 78.13$ Para el grupo B (hidrocortisona) tenemos, en los mismos parámetros: Edad, rango entre 28.07 años con M+DE 56.03; peso entre $54.14 \mathrm{~kg}$ y $M+D E$ 76.06; talla comprendida entre $148.28 \mathrm{cms}$ con M+DE de 162.92 y duración de intervención quirúrgica con rangos entre 36.57 minutos y $M+D E$ de 101.43 Para el grupo C (dexametasona) se encuentra que la edad oscila entre 29.04 años con M+DE 57.96; el peso entre 56.03 kilos con M+DE 79.17; talla entre $149.13 \mathrm{cms}$ con M+DE 162.25 y tiempo de duración de entre 41.93 min. Con M+DE de 78.07 Observándose que estadísticamente, al no haber diferencias significativas entre los grupos; no estaría influenciada la aparición de CPPD.

En el siguiente gráfico se observa la relación de la edad, tomando en cuenta la M+DE para cada uno de los grupos; observándose de manera explícita las similitudes de los grupos. En el grupo A 44.65; el grupo B 42.05 y el grupo C 43.5

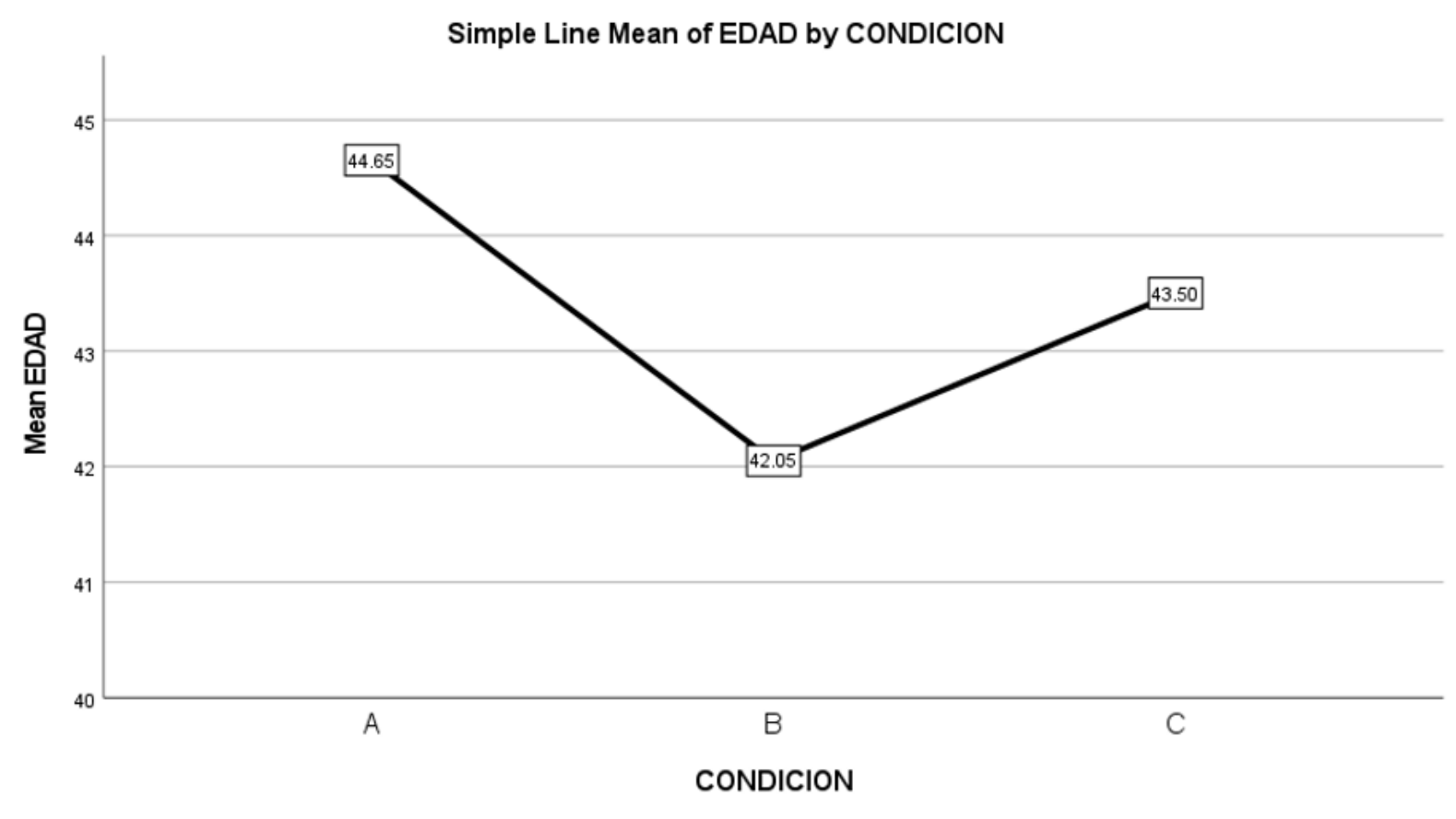

Error Bars: $95 \% \mathrm{Cl}$

Gráfico 1. Medias de Edades en cada grupo

El próximo gráfico se observa la relación con respecto al peso en cada uno de los grupos, observándose la similitud en dichos grupos. En el grupo A se obtuvo un valor de 68.1; en el grupo B se cuenta con una media de 65.1; y en el grupo C 67.6. 


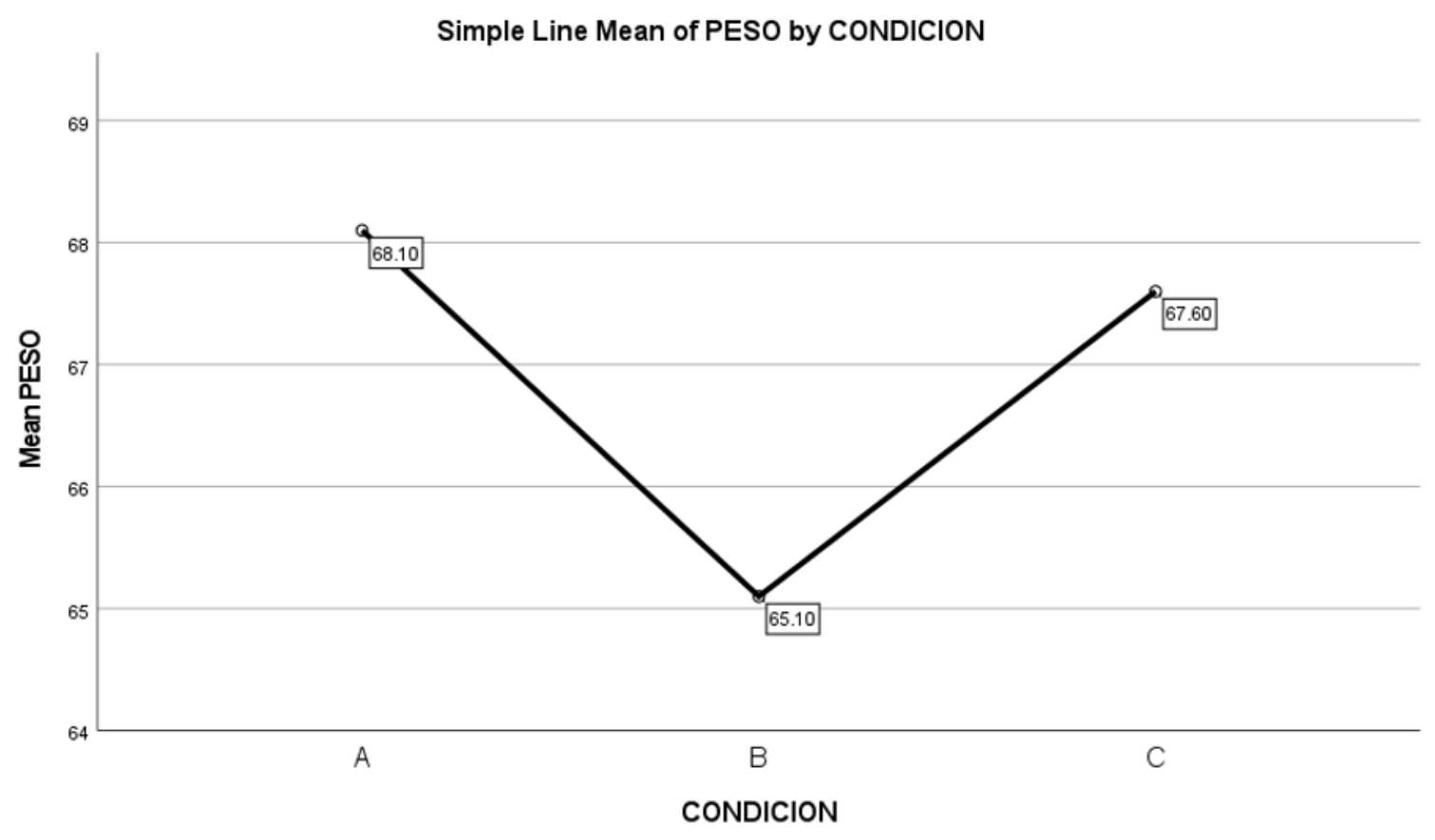

Gráfico 2. Medias de Peso en cada grupo

Como en los anteriores, se anexa el gráfico número 3; en donde se comparan las medias entre las tallas de las pacientes de cada grupo. En el grupo A se tiene una media de 156.55; el grupo B cuenta con 155.6 y en el grupo C.- 155.95.

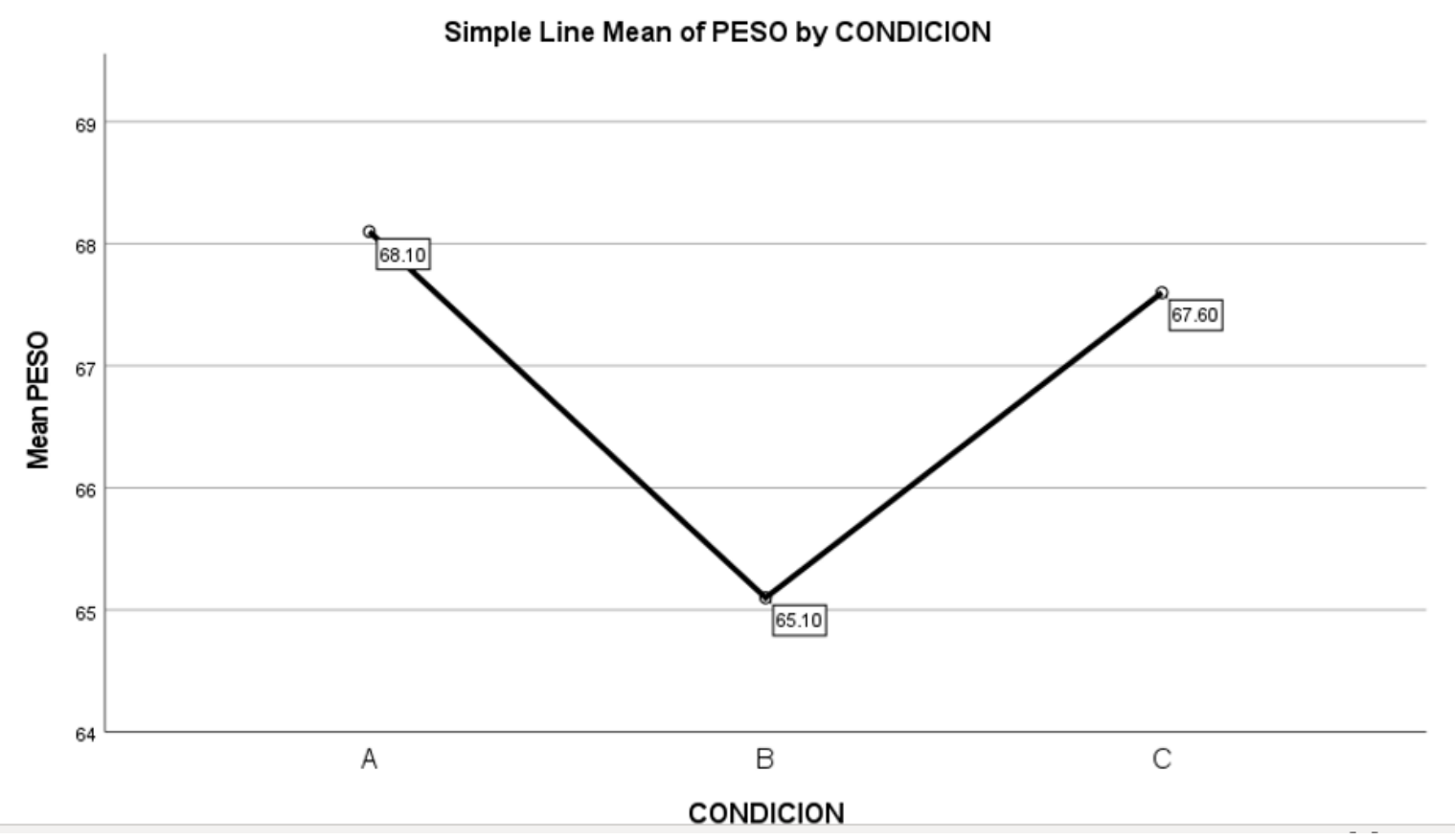

Gráfico 3. Medias de la Talla en cada grupo 
En el último gráfico, el $\mathrm{N}^{\circ} 4$ se observa la media del tiempo quirúrgico en cada uno de los grupos, en el grupo A se obtuvo una media de tiempo de $61.5 \mathrm{~min}$; en el grupo B 69 y en el grupo C 60.

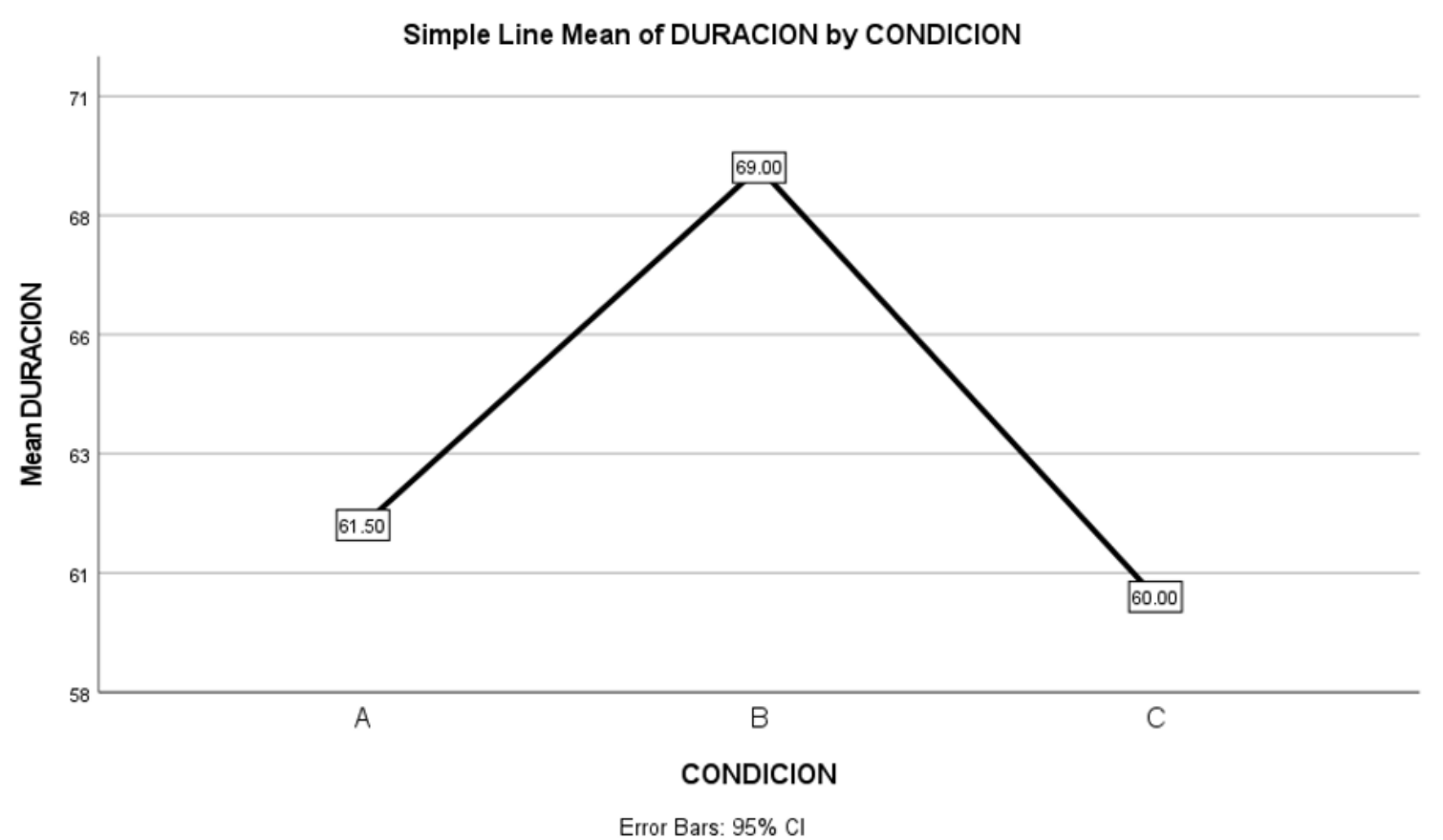

Gráfico 4. Medias del Tiempo Quirúrgico en cada grupo.

En cuanto a los síntomas relacionados con CPPD, se expone en la siguiente tabla, detalladamente, cada síntoma y se indican en qué grupo estuvo presente y expresado en números absolutos las pacientes quienes lo presentaron. En el grupo A, en grupo control, se observó que la cefalea ortostática, cefalea bilateral, rigidez nucal, fotofobia, diplopía, náuseas, vómitos y realización previa de punción lumbar fueron presentados por dos pacientes, mientras que el tinnitus y los mareos, estuvieron presentes solo en 1 paciente. En el grupo B, utilizando Hidrocortisona, no se presentaron síntomas de CPPD y en el grupo C, utlizando dexametasona, tampoco se observaron los síntomas de cefalea ortostática, con la realización previa de punción lumbar.

Tabla 2. Número de pacientes con presencia de síntomas de CPPD.

\begin{tabular}{lccc}
\hline SíNTOMAS & GRUPO A & GRUPO B & GRUPO C \\
\hline Cefalea Ortostática & 2 & 0 & 0 \\
Cefalea Bilateral & 1 & 0 & 0 \\
Rigidez Nucal & 1 & 0 & 0 \\
Tinnitus & 1 & 0 & 0 \\
Hipoacusia & 1 & 0 & 0 \\
Hiperacusia & 0 & 0 & 0 \\
Fotofobia & 2 & 0 & 0 \\
Diplopia & 1 & 0 & 0 \\
Náuseas & 2 & 0 & 0 \\
Vómitos & 1 & 0 & 0 \\
Mareos & 1 & 0 & 0 \\
Realivación previa de punción lumbar & 1 & 0 & 0 \\
Remisión espontánea & 2 & 0 & 0 \\
\hline
\end{tabular}

Fuente: Calle (2018) 
Tabla 3. Porcentaje de pacientes con presencia de síntomas de CPPD.

\begin{tabular}{l|ccc|} 
SÍNTOMAS & GRUPO A & GRUPO B & GRUPO C \\
\hline Cefalea Ortostática & $10 \%$ & $0 \%$ & $0 \%$ \\
Cefalea Bilateral & $5 \%$ & $0 \%$ & $0 \%$ \\
Rigidez Nucal & $5 \%$ & $0 \%$ & $0 \%$ \\
Tinnitus & $5 \%$ & $0 \%$ & $0 \%$ \\
Hipoacusia & $5 \%$ & $0 \%$ & $0 \%$ \\
Hiperacusia & $0 \%$ & $0 \%$ & $0 \%$ \\
Fotofobia & $10 \%$ & $0 \%$ & $0 \%$ \\
Diplopía & $5 \%$ & $0 \%$ & $0 \%$ \\
Náuseas & $10 \%$ & $0 \%$ & $0 \%$ \\
Vómitos & $5 \%$ & $0 \%$ & $0 \%$ \\
Mareos & $5 \%$ & $0 \%$ & $0 \%$ \\
Realización previa de punción lumbar & $5 \%$ & $0 \%$ & $0 \%$ \\
Remisión espontánea & $10 \%$ & $0 \%$ & $0 \%$
\end{tabular}

Fuente: Calle (2018)

En la siguiente tabla, se observa el tiempo de aparición de CPPD posterior a la aplicación de la técnica anestésica. Observándose en el Grupo A, que las dos pacientes que presentaron CPPD, se iniciaron los síntomas posterior a las 24 horas, específicamente a las 19 y 28 horas respectivamente y los pacientes de los grupos B y C, no presentaron síntomas.

Tabla 4. Tiempo de aparición de CPPD

\begin{tabular}{lccc}
\hline & Grupo A & Grupo B & Grupo C \\
\hline Tiempo de & 19 & - & - \\
aparición (horas) & 28 & - & -
\end{tabular}

Fuente: Calle (2018)

En la siguiente tabla, se observa el tiempo En ninguno de los tres grupos se observaron reacciones adversas a la técnica, anestésica ni a los medicamentos aplicados.

\section{Discusión}

En la tabla 4 se exponen las variables propias de los pacientes e independientes como lo son edad, peso, talla y tiempo quirúrgico, las cuales podrían influenciar en la aparición o no de CPPD; sin embargo, como se puede observar, las diferencias entre los grupos no son estadísticamente significativas, teniendo una distribución normal de los datos, por lo tanto se puede inferir y concluir $(p>0,05)$ que estos parámetros no influenciarían en la incidencia de
CPPD, permitiendo controlar esta variable a disposición del investigador y precisamente dando grados de libertad para la aplicación de la variable de estudio, que es la utilización de la profilaxis para la CPPD.

La literatura, como se ha dicho ya, relaciona algunos tipos de aguja para punción raquídea con mayor o menor incidencia de CPPD, por lo cual se decidió controlar también este parámetro, usando el mismo tipo de aguja (Sprotte) y calibre (25G), en todos los pacientes; tomando en cuenta que la aguja tipo Sprotte, es la menos relacionada con la aparición de CPPD relacionada al tipo de aguja, con lo cual se descarta su influencia en la aparición de la misma, así como el calibre $25 \mathrm{~g}$, que aunque es de un 
grosor que podría relacionarse con aparición de CPP, al ser usado en todos los pacientes de la muestra se tiene control sobre esta variable, descartándola como un parámetro que podría alterar los resultados de la investigación.

Los síntomas relacionados con CPPD aparecidos en este estudio fueron la cefalea ortostática, fotofobia y náuseas, cefalea bilateral, rigidez nucal, tinnitus, hipoacusia, diplopía, vómitos, mareos y realización previa de punción lumbar; todos, relacionados directamente con la CPPD y asociados como los más frecuentes en aparición a través de los distintos estudios internacionales y literatura citada; por lo cual, dichos resultados, podrían sin duda, determinar que se tiene un diagnóstico certero de CPPD y por lo tanto su aparición o no, podría relacionarse directamente a la efectividad de la profilaxis realizada.

Aunque la incidencia de CPPD, es baja tanto en valores absolutos como porcentuales, se observa significativamente que ningún paciente en el Grupo B (profilaxis con hidrocortisona 500mg IV), ni tampoco ningún paciente del grupo $\mathrm{C}$ (profilaxis con dexametasona $16 \mathrm{mg}$ IV) presentaron CPPD, no así en el grupo control donde se observó la presencia de CPPD e dos pacientes.

Cabe de esta forma destacar que podemos afirmar que hay diferencia entre los pacientes de los grupos $\mathrm{B}$ y $\mathrm{C}$ que recibieron profilaxis para CPPD, con los pacientes del Grupo A, grupo control. De la misma forma, que no habiendo diferencia entre los pacientes del Grupo B con los pacientes del Grupo C, la eficacia de profilaxis con hidrocortisona y dexametasona, en este estudio en particular es la misma en la prevención de la CPPD.

Según los resultados del estudio, la aparición de CPPD fue entre las 19 y 28 horas, coincidiendo con lo que la literatura internacional ha acordado con respecto a este tema, situando al estudio actual, como efectivo al relacionarse con los resultados obtenidos en otros estudios.

Aunque estadísticamente, no se cuente con mucha diferencia porcentual y significativa entre los tres grupos; tomando en cuenta las bases médico legales y carácter biopsicosocial de dichos casos descritos; son significativamente determinantes y por lo tanto debería instaurarse la conducta de profilaxis de CPPD con los esteroides estudiados como protocolo en la aplicación de anestesia por punción raquídea.

\section{Conclusiones}

- Las variables edad, peso, talla, tiempo quirúrgico, no influencian ni alteran los resultados obtenidos en el estudio, debido a que no hay diferencias significativas en las mismas. Así mismo, las variables de tipo de aguja y calibre de la misma, al ser la misma en todos los pacientes, tampoco altera el resultado final del estudio, comprobando su confiabilidad.

- La incidencia de CPPD en este estudio fue del $10 \%$ en el grupo control y de $0 \%$ en el grupo experimental con hidrocortisona y dexametasona; la cual, considerando algunas series internacionales, publicadas al respecto, (de 40 a 60\%) se encuentra por debajo de lo publicado; pudiendo indicar, que la profilaxis fue efectiva en reducir la totalidad de la incidencia global de CPPD en las pacientes.

- Los síntomas relacionados con la CPPD, resultaron exactamente iguales a los expuestos internacionalmente; el cuadro de CPPD y sus síntomas están bien tipificados y definidos, no se observó algún síntoma relevante o diferente asociado al cuadro de CPPD en este estudio.

- El tiempo de aparición de la CPPD osciló entre las 19 y 28 horas después de la punción, también dentro de lo esperado con respecto a la estadística in-

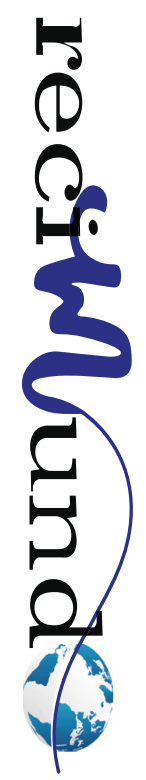


ternacional ya descrita; algunas series las describen entre las primeras 24 a 72 horas, otras a partir de las 72 horas e incluso algunos estudios reportan casos de CPPD después de semanas o meses posterior a la punción anestésica, lo que no ocurrió en nuestro estudio.

- Las diferencias en incidencia de CPPD entre los dos grupos experimentales (B con hidrocortisona y $\mathrm{C}$ con dexametasona) no existen; lo que es opuesto a la diferencia de los grupo de pacientes $B$ y $C$ con los del grupo $A$; esto es significativamente valioso para concluir de la utilidad del uso indistinto de hidrocortisona o dexametasona con efectos útiles para la prevención de CPPD en pacientes que son tratados con anestesia raquídea.

\section{Recomendaciones}

- Aplicar profilaxis contra CPPD en pacientes que reciban anestesia raquídea, dado la posibilidad real de presentar esta complicación.

- Profundizar el estudio y extenderlo a otros grupos epidemiológicos, así como también determinar con exactitud qué factores modificables (como tipo de aguja, calibre de la aguja, nivel del bloqueo, técnica de punción, o patología presente en el paciente a anestesiar) son asociados más frecuentemente a CPPD en la población ecuatoriana.

- Extender el nivel del estudio, y hacerlo multicéntrico, para lograr una visión extendida sobre la epidemiología de la CPPD en nuestra población.

- Comparar esta profilaxis propuesta en el estudio con otras profilaxis aplicadas en otros estudios y así extender el nivel de conocimiento sobre CPPD y formas de evitarla.

- Exponer en conferencias, pósters, mesas redondas y ponencias en congresos, los resultados de este estudio y compararlos con otros similares a nivel nacional e internacional.

\section{Bibliografía}

Línea y Salud. (15 de julio de 2021). Diccionario digital de definiciones de términos médicos. Obtenido de www.lineaysalud.com: https://www.lineaysalud. com/que-es/aine

López Correa, T., Garzón Sánchez, J., Sánchez Montero, F., \& Muriel Viloria, C. (2011). Cefalea postpunción dural en obstetricia. Revista Española de anestesiología y reanimación, 563-573.

Marrón Peña, M., \& Mille Loera, J. (2013). Realidades terapéuticas de la cefalea postpunción dural. Revista Mexicana de Anestesiología, 277-282.

Moral Turiel, M., Rodríguez Simón, M., Sahagun de la Lastra, J., \& Yuste Pascual, J. (2002). Tratamiento de la cefalea post punción dural con hidrocortisona intravenosa. Revista Española de Anestesiología y Reanimación, 101-104.

Oceano-Mosby. (2001). Diccionario de términos médicos. México: Oceano Mosby.

Rendón Mejías, A. (2002). Seguridad en anestesia. Anestesia ginecoobstétrica. Revista Mexicana de Anestesiología, 240-248.

Torres Zafra, J. (2008). Cefalea postpunción lumbar . Acta Neurológica Colombiana, 112-117. 


\section{CITAR ESTE ARTICULO:}

Calle Gómez, M. A., Devonish, N., Gurumendi España, I. E., \& Borja Santillán, M. A. (2021). Dexametasona o hidrocortisona intravenosa como profilaxis de cefalea postpunción dural. RECIMUNDO, 5(3), 52-65. https://doi.org/10.26820/ recimundo/5.(2).julio.2021.52-65 